Research Paper

\title{
In vivo Antitumor Effect of an HPV-specific Promoter driving IL-12 Expression in an HPV 16-positive Murine Model of Cervical Cancer
}

\author{
Victor Hugo Bermúdez-Morales, ${ }^{1}$ Geny Fierros-Zarate, ${ }^{1}$ Celina García-Meléndrez, ${ }^{2}$ Juan Manuel \\ Alcocer-Gonzalez, ${ }^{3}$ Ausencio Morales-Ortega, ${ }^{1}$ Oscar Peralta-Zaragoza, ${ }^{1}$ Kirvis Torres-Poveda, ${ }^{1}$ Ana Isabel \\ Burguete-García, ${ }^{1}$ Eva Hernández-Márquez, ${ }^{1}$ Vicente Madrid-Marina ${ }^{1 凶}$ \\ 1. Division of Chronic Infection and Cancer, National Institute of Public Health. Av. Universidad 655, Cuernavaca, Morelos. México. 62100. \\ 2. Biotecnhgology Institute. National Autonomous University of Mexico, Cuernavaca Morelos, México. \\ 3. School of Biological Sciences, Autonomous University of Nuevo León, Monterrey, México.
}

$\triangle$ Corresponding author: Vicente Madrid Marina. Division of Chronic Infections and Cancer, National Institute of Public Health, Av. Universidad 655, Col. Santa María, Ahuacatitlán. Cuernavaca, Morelos. México. 62100. Tel: (+52-777) 329-30-56 Fax: (+52-777) 317-54-85 Email: vmarina@insp.mx.

(C) Ivyspring International Publisher. Reproduction is permitted for personal, noncommercial use, provided that the article is in whole, unmodified, and properly cited. See http://ivyspring.com/terms for terms and conditions.

Received: 2016.03.14; Accepted: 2016.06.29; Published: 2016.09.27

\begin{abstract}
Human papillomavirus (HPV) is a DNA virus that infects epithelial cells and has been implicated in the development of cervical cancer. Few therapeutic strategies have been designed for the treatment of cervical intraepithelial neoplasia, a precursor of cervical cancer. In these early stages, the HPV E2 protein is the most important viral factor involved in viral gene expression and plays crucial roles during the vegetative viral cycle in epithelial cells. Papillomavirus E2 binds specifically to palindromic $A C C N_{6} G G T$ sequences, referred to as the E2 binding sites (E2BS), which are concentrated within the viral long control region, and which are responsible for regulation of the HPV protein's expression. Here, we consider E2BS as a candidate sequence to induce the expression of antiviral therapeutic genes selectively in HPV-infected cells expressing the E2 protein. This study focuses on the use of an HPV-specific promoter comprised of four E2BS to drive the expression of IL-12, leading to an antitumor effect in an HPV-positive murine tumor model. The therapeutic strategy was implemented via viral gene therapy using adenoviral vectors with recombinant E2 and IL-12 genes and E2BS-IL-12. We demonstrate that the HPV-specific promoter E2BS is functional in vitro and in vivo through transactivation of HPV E2 transcription factor.
\end{abstract}

Key words: HPV, promoter, IL-12, gene therapy, adenovirus, HPV E2, antitumor.

\section{Introduction}

Cervical cancer (CC), which is associated with persistent infection with high-risk human papillomavirus (HPV), is one of the most important neoplasias in global public health ${ }^{1}$. The high incidence of mortality and morbidity caused by this cancer, exceeded only by breast cancer in some populations ${ }^{2,3}$, warrants special attention toward the development of new strategies for control and treatment at early stages to prevent disease evolution.

One approach that has been extensively investigated is gene therapy 4,5 , which has been shown to have antitumor activity through induction of cell death. Most gene therapy studies have been based on therapeutic genes under the control of ubiquitous promoters such as CMV, SV40, heat shock, etc., which induce low specific toxicity in normal cells and tissues, as well as in cancer cells ${ }^{6,7}$. The targeted expression of therapeutic genes is essential to minimize the toxic effects due to the use of the recombinant protein $7-10$ and to maximize the therapeutic effect. One gene therapy approach that has been used to target HPV-cancer cells in murine 
tumor models takes advantage of the HPV16 Long Control Region (LCR) DNA sequence to manipulate gene expression in HPV-infected cells and uninfected cells. However, the LCR of HPV is activated in both HPV16-infected cells and non-infected cells ${ }^{11,12}$. Nevertheless, other HPV-specific sequences that are controlled only by HPV proteins may have applications in therapies for precancerous lesions and cervical cancer.

The HPV E2 proteins have the same functions in the more than 100 characterized papillomavirus genotypes and are key regulators of the viral life cycle ${ }^{13,14}$. E2 protein functions are mediated through protein-protein interactions and through the binding of E2 to a palindromic DNA sequence ACCN 6 GGT, referred to as the E2 binding site (E2BS) ${ }^{15-17}$. E2BS merits consideration as a candidate sequence to drive the expression of therapeutic genes only in HPV-infected cells that express the HPV E2 protein. The construction of an HPV-specific promoter to induce expression of immunoregulatory cytokines, such as IL-12, is a promising approach with potential applications in the treatment of precancerous lesions before HPV integration.

Interleukin 12 (IL-12) is a cytokine with multiple immune functions, including the stimulation of antitumor immune responses, but it has not yet become a standard therapeutic agent in clinical care ${ }^{18}$. Numerous preclinical studies demonstrated the benefit of IL-12 in several different tumor models, and these positive results led to clinical trials ${ }^{19-24}$. In cervical cancer a predominance of the Th2-type cytokine profile is observed in association with a diminished Th1-type cytokine profile ${ }^{25-28}$. The shift to Th2-type cytokines during the course of cervical cancer development is reflected in an increased serum concentration of Th2-type cytokines while concentration of the tumor-infiltrating T-lymphocytes decrease as the lesion progresses $28-30$. Therefore, upregulation of cytokines such as IL-12, which favors the development of Th1 cells, through an HPV-specific promoter represents a promising approach to induce activation of the immune response in precancerous lesions, as well as to inhibit Th2-type cytokines, in order to prevent progression of the malignancy.

The objective of this study was to utilize an HPV-specific promoter comprised of four E2BS's to induce expression of IL-12 and produce antitumor effects in an HPV-positive murine tumor model. The therapeutic strategy was implemented through viral gene therapy using adenoviral vectors with recombinant E2, IL-12 genes and E2BS-IL-12. We demonstrated that the HPV specific-promoter E2BS was functional in vitro and in vivo due to transactivation by the HPV E2 transcription factor.

\section{Materials and Methods}

Cell Culture. The BMK-16/myc murine cell line was kindly donated by Dr. Sophie Hallez (Université Libre de Bruxelles, Rhode-Saint-Genèse, Belgium). This cell line was established by co-transformation of baby BALB/c kidney cells with the c-myc gene and the HPV 16 genome, as previously described ${ }^{31}$. The C-33 A (ATCC) and AD293 (Stratagene, Calif., USA) were grown in a DMEM medium (Invitrogen, Carlsbad, Calif., USA) and supplemented with 10\% fetal bovine serum, penicillin/streptomycin (50 $\mathrm{ug} / \mathrm{ml}), 2 \mathrm{mM}$ L-glutamine and $250 \mathrm{ng} / \mathrm{ml}$ fungizone (Invitrogen) at $37^{\circ} \mathrm{C}$ in $5 \% \mathrm{CO}_{2}$.

Plasmids. The E2-pCMVp16 plasmid (which contains the open reading frame (ORF) of the gene encoding the HPV16 E2 protein) and pC18SP1Luc plasmid (which contains four response elements to the E2 protein of HPV16 and a SP1 luciferase reporter gene site) were donated by Dr. G Veress (School of Medicine, University of Debrecen, Hungary $)^{32}$ The plasmid pNGVL3-mIL-12, which contains clones of the two subunits (p35 and p40) of mouse. Plasmids were propagated in DH5- $\alpha$ bacteria and were purified by alkaline lysis; integrity was verified on $0.8 \%$ and $1 \%$ agarose gel.

Construction of Recombinant Adenovirus. Using the AdEasy system, recombinant bacterial adenovirus was generated with defective replication. The pAdtrack-CMV plasmid was used to clone the IL-12 gene with the cytomegalovirus promoter (CMV) as the promoter sequence. The plasmid pAdTrack was used to clone the IL-12 gene with 4 E2 binding sites (E2BS) as the promoter sequence. Briefly, the recombinant adenovirus AdCMVmIL-12 was generated by cleaving the two subunits (p35 and p40) of the IL-12 gene from the plasmid pNGVL-3-mIL-12 at the restriction sites $B g l I I$ and $E c o R V$. The gene was cloned into these same sites in the IL-12 pAdTrack-CMV vector, and clones were selected with kanamycin. The adenoviral vector AdE2mIL-12 expressing IL-12 under control of the four E2BS as its promoter sequence was generated as follows. The E2Sp1 promoter was cleaved at the sites BamH I/ Hind III from the plasmid pC18SP1Luc and was cloned into the pCDNA3 vector to be sequenced and was sub-cloned into the pAdTrack vector at the Hind $I I I / E c o R V$ sites. The vector generated, pAdTrack SP1E2, was cloned into the EcoR $V$ and $B g l ~ I I$ sites and the two subunits of IL-12 were released at the same sites of the aforementioned pNGVL3-mIL-12 construction. Thus the pAdTrack-E2mIL-12 plasmid was generated and the poly-adenylation sequence was amplified by PCR from the original vector 
mIL-12, and was cloned into the unique $B g l$ II site (pAdTrack-E2mIL-12), with orientation verified by PCR. The plasmids generated were recombined with the plasmid pAdenoEasy 1 in BJ5183 E. coli to generate a recombinant plasmid. Finally, recombinant plasmids were cut with the restriction enzyme Pac I and transfected into AD293 cells to generate the adenoviruses AdCMV, AdCMVmIL-12 and AdE2mIL-12. Virion production was monitored by detection of the green fluorescent protein (GFP) produced by the virus, which was visualized by vertical epifluorescence microscopy. Cells were harvested in the presence of $5 \mathrm{~mL}$ of buffer A $(10 \mathrm{mM}$ Tris HCL, $0.5 \mathrm{M} \mathrm{NaCl}$, $\mathrm{pH}$. 8.0), then were centrifuged at 3,000 rpm and lysed (by heat and cold). The lysate was centrifuged at 3,000 rpm for $5 \mathrm{~min}$ at $4^{\circ}$ $\mathrm{C}$, and the supernatant was used for large-scale production of recombinant adenoviruses. T75 twenty culture dishes with $100 \%$ confluence of AD293 cells were infected with $1 \mathrm{~mL}$ of the supernatant. Five days after infection the cells were harvested with shaver (scraper) in the presence of $10 \mathrm{ml}$ of buffer A. The cells were lysed by heat and cold and centrifuged at 3,000 rmp. Virions were purified from the supernatant by $\mathrm{CsCl}$ gradient ultracentrifugation $(32,000 \mathrm{rpm}, 18 \mathrm{~h}$ at $4^{\circ} \mathrm{C}$ ), dialyzed and stored at $-70^{\circ} \mathrm{C}$. Viral titers (PFU) were determined by plaque assays in AD293 cells according to the protocol of Vogelstein ${ }^{33}$ Western blot analysis was used to verify production of E2 and IL-12 protein in AD293 and BMK-16/myc cells. Additionally, we generated an adenovirus expressing GFP (AdCMVGFP) as a control.

Tumor growth inhibition assay. Tumor model: preclinical evaluation was carried out in an HPV 16-positive murine tumor model. ${ }^{34} 5 \times 10^{5} \mathrm{BMK}-6 /$ myc cells were injected subcutaneously into the back of $\mathrm{Balb} / \mathrm{c}$ mice at a previously shaved site. Each group of five mice (provided by the animal facility of the National Institute of Public Health, strain from Charles River Laboratories, Wilmington, MA, USA), had their tumor volume measured in $\mathrm{mm}^{3}$ every 3 days $\left(\mathrm{V}=\right.$ larger diameter $\mathrm{X}$ smaller diameter $\left.{ }^{2}\right) / 2$ and their survival was reported. Tumor volume was studied to determine the therapeutic effect of the recombinant adenovirus. Tumor volume of 20 to 30 $\mathrm{mm}^{3}$ was considered time zero and the subsequent effect of adenovirus on tumor growth and mouse survival was measured. Administration of recombinant adenovirus was as follows: each group of five mice with a tumor volume of $20-30 \mathrm{~mm}^{3}$ had $5 \times 10^{5}$ PFU of recombinant virions containing either AdCMVE2HPV16, AdCMVmIL-12 or AdCMVGFP, administered intra-tumorally in a final volume of 30 $\mathrm{ul}$ in 1X PBS. Another group of five mice had $5 \times 10^{3}$ PFU of each adenovirus (AdCMVE2HPV16,
AdE2mIL-12) co-administered intra-tumorally in a final volume of $30 \mathrm{uL}$. All mice were monitored for a period of 28 days; tumor inhibition was determined by measuring tumor volume $\left(\mathrm{mm}^{3}\right)$ every three days with a digital caliper and survival curve.

Western Blot Assay. BMK-16/myc cells, C-33 A and AD293 were harvested at $48 \mathrm{~h}$ after the infection with recombinant adenovirus and were lysed in cold RIPA lysis buffer $(20 \mathrm{mM}$ Tis- $\mathrm{HCl}, \mathrm{pH} 7.4,10 \mathrm{mM}$ $\mathrm{NaCl}, 10 \mathrm{mM} \mathrm{KCl}, 3 \mathrm{mM} \mathrm{MgCl} 2,0.5 \%$ triton X-100, $0.5 \%$ NP-40 and protease inhibitors). The proteins were then collected by centrifugation. Protein concentrations were determined using the BCA protein assay kit (Pierce, Rock- ford, Ill., USA), according to the manufacturer's instructions. Approximately $50 \mathrm{ug}$ of total protein were electrophoresed in SDS-PAGE 10\% and the resolved proteins were transferred to nitrocellulose membranes (Amersham Biosciences, Piscataway, N.J., USA). The blots were blocked in 5\% dry non-fat milk in TBS (20 mM Tris-base, $\mathrm{pH} 7.6,137 \mathrm{mM} \mathrm{NaCl}$ and 1 $\mathrm{M} \mathrm{HCl}$ ) with $0.05 \%$ Tween-20 for $2 \mathrm{~h}$. Later, the membrane was washed with PBS 1X and incubated with the appropriate antibody for $16 \mathrm{~h}$ at $4^{\circ} \mathrm{C}$. The membrane was incubated with the secondary antibody for 30 to $45 \mathrm{~min}$ at room temperature. The detection was carried out using ECL Western Lightning Chemiluminescence Reagent Plus (PerkinElmer Life Sciences, Boston, Mass., USA). The antibodies were diluted in $2.5 \%$ dry non-fat milk in Tween-20 TBS solution. The antibody dilutions used were anti-actin (dilution 1:1,000), anti-IL-12, p40, (dilution 1:500), anti-goat HRP (dilution 1:10,000) and anti-mouse (dilution 1:8,000; Santa Cruz Biotechnology, Santa Cruz, Calif., USA).

Immunohistochemistry. Biopsies of tumor-bearing mice treated intratumoral with the recombinant adenovirus were paraformaldehydefixed. Serial frozen tissue sections were cut into $10 \mu \mathrm{m}$ generated by transversal cryo-sections fixed in acetone and methanol for $10 \mathrm{~min}$ and washed in PBS for $3 \mathrm{~min}$ at room temperature. After the tissues were blocking for 30 min with 5\% BSA and wash in PBS $0.2 \%$ Tween 4 times for $5 \mathrm{~min}$, and were permeabilized with $0.2 \%$ Triton for $10 \mathrm{~min}$. The incubation of the primary antibodies was done overnight at $4^{\circ} \mathrm{C}$, follow with a wash in PBS $0.2 \%$ Tween 4 times for $5 \mathrm{~min}$ at room temperature. Slides were then incubated with conjugated secondary antibody $30 \mathrm{~min}$ to $1 \mathrm{hr}$ at room temperature. Fluorescence was visualized with Zeiss Axioskop Epifluorescencet microscope (Zeiss, Germany). Images were acquired using a Micro-ManagerExaminer 1.2, 20X. The antibody dilutions anti-IL-12 p40 (dilution 1:500), serum anti-HPV16 E2 protein 
(dilution 1:250) ${ }^{34}$ and conjugated secondary antibody anti-goat Cy3 (1:1000) for IL-12 and Anti-mouse (1:1000) by HPV 16 E2 (Jackson ImmunoResearch LABORATORIES, INC. (West Grave, PA.USA).

\section{Statistical Analysis}

Data were analyzed and provide average $(X)$ and standard error (s). Additionally, an analysis of Student's t-test STATA 9.1., was done to determine differences between the experimental groups. A P value of $\geq 0.05$ significance values was used for all statistical tests.

\section{Results}

Generation of recombinant adenovirus. AD293 cells were infected with $50 \mathrm{MOI}$ of the recombinant adenovirus: AdCMVGFP, AdCMVE2HPV16, AdCMVmIL-12 and AdE2mIL-12 for large-scale production and the virions was monitoring by the detection of GFP expression. As shown figure 1, GFP expression was visible $48 \mathrm{hr}$ after the infection in $80-90 \%$ of the cells showing cell rounding and small amounts of clumping with cytopathic effects.

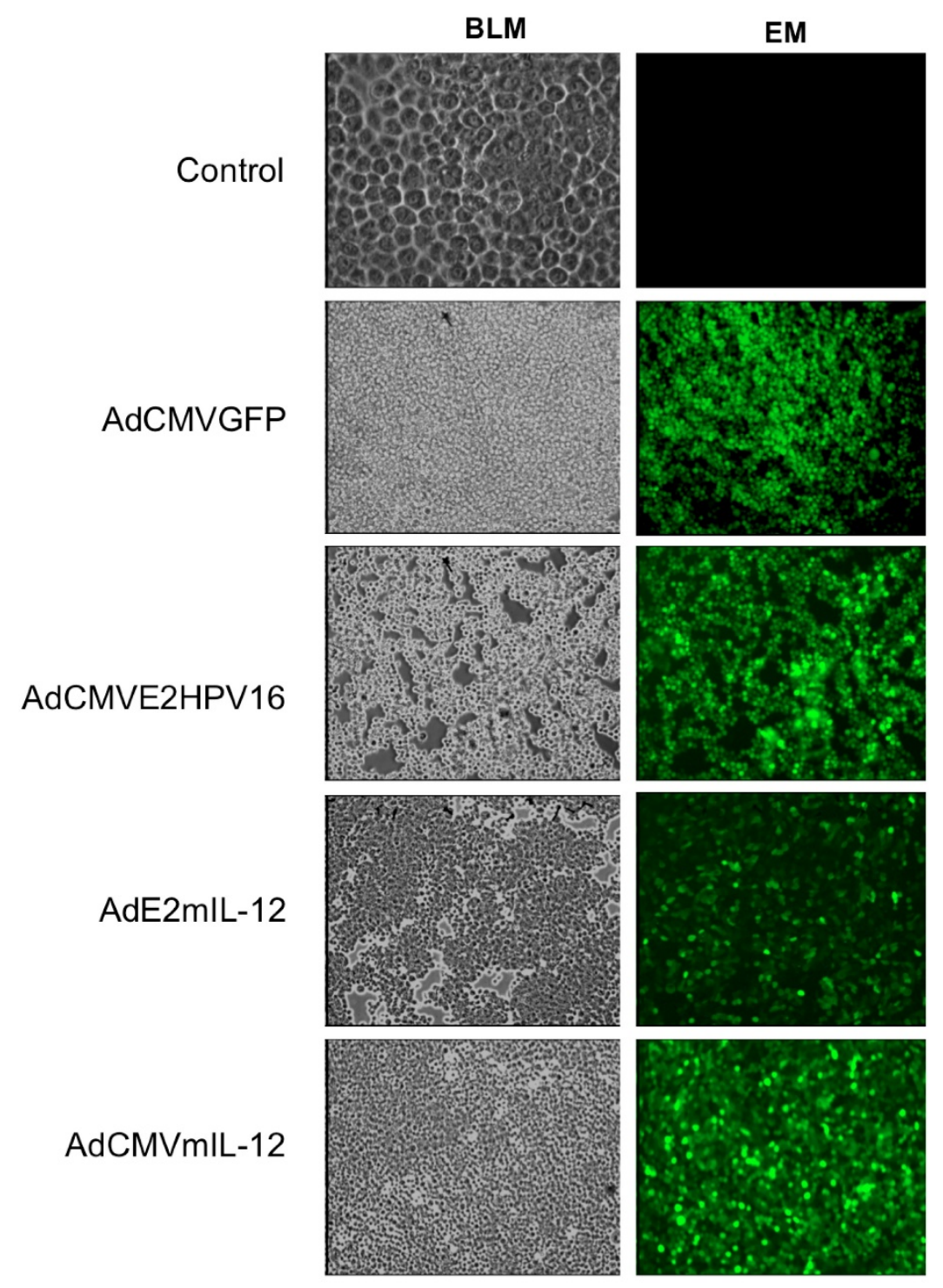

Figure 1: Production of recombinant adenovirus monitored by GFP expression. AD293 cells transfected with 50 MOI of each recombinant adenovirus (AdCMVGFP, AdCMVE2HPV16, AdCMVmlL-12 and AdE2mlL-12); $24 \mathrm{hr}$ later the cells infected were observed on Epifluorescence microscopy and the expression of GFP protein was monitored. The cells showed cell rounding and small amounts of clumping with cytopathic effects. Control: AD293 cells no infected. 
A

$\begin{array}{lllll}0 & 50 & 100 & 500 & \text { MOI AdCMVmIL-12 }\end{array}$
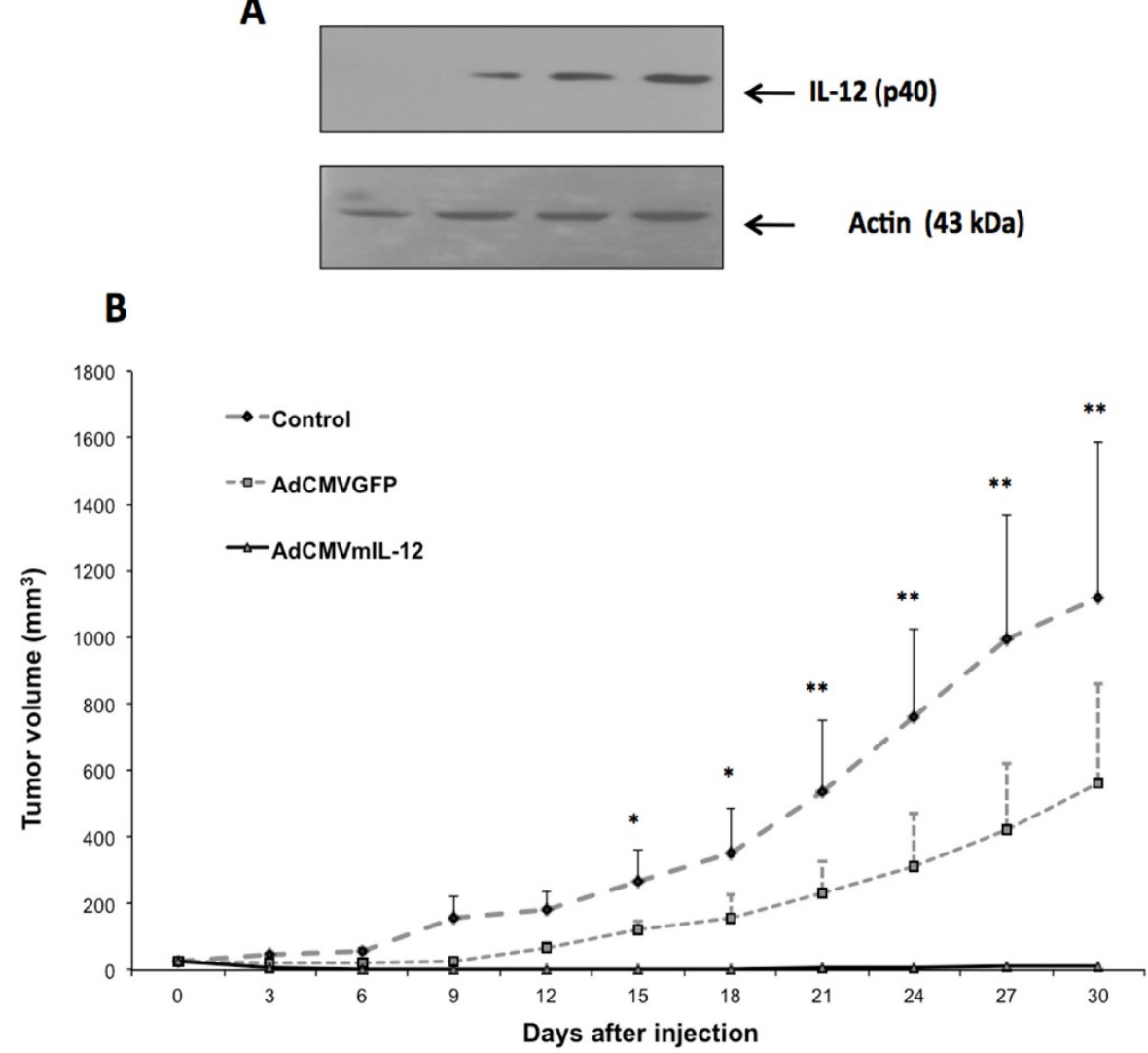

Figure 2: Antitumor effect of recombinant adenovirus AdCMVIL-12 in a murine tumor model. A). Detection of IL-12 protein by western blot BMK-16 / myc cells infected with adenovirus AdCMVmIL-12. B). Antitumor effect of AdCMVmlL-12 treatment compared to AdCMVGFP treatment compared to no treatment. Data are presented as $X \pm S E$. *P <0.05: AdCMVmlL-12 and AdCMVGFP versus controls. **P <0.01: AdCMVmlL-12 and AdCMVGFP versus controls (Student's t-test)

Expression of AdCMVIL-12 in vitro and antitumor effect in vivo. In BMK-16/myc cells transduced with AdCMVmIL-12, we detected the p40 subunit of IL-12, which has a molecular weight of 40 $\mathrm{kDa}$, by Western Blot (Fig. 2A); IL-12 p40 was not detected in uninfected cells. Detection of p40 protein was dose-dependent and correlated with the MOI of AdCMVmIL-12 with which epithelial cells were infected. We found that intratumoral injection of AdCMVmIL-12 into tumor-bearing mice was associated with a decrease in tumor size over time. In $80 \%$ of mice almost complete resolution of tumor was observed and this was maintained up to 30 days after injection (Fig. 2B). In contrast, the tumor-bearing mice injected with AdCMVGFP showed only a slight reduction in tumor growth, and continued to have sustained growth, while the untreated mouse controls showed continuous growth of tumor.

In vitro and in situ activity of an HPV-specific promoter in epithelial cells. Prior to its application in the in vivo tumor model, we evaluated the HPV-specific promoter comprised of four E2 protein-binding sites which induces IL-12 expression using recombinant adenovirus AdCMVE2HPV16, which expresses the HPV 16 E2 protein. ${ }^{18}$
BMK-16/myc cells were co-infected with AdE2mIL-12 (50 MOIs) and increasing concentrations of AdCMVE2HPV16 and was detected the E2 protein. Induction of the $\mathrm{p} 40$ subunit of IL-12 was detected at a concentration of $10 \mathrm{MOIs}$ of the recombinant adenovirus AdCMVE2HPV16, but detection of p40 subunit of IL-12 was more evident at 50 and 100 MOIs after AdCMVE2HPV16 infection (Fig. 3A). These results demonstrate the functional activity of this HPV E2-specific promoter in an in vitro system. Addition, the in vitro activity of the HPV E2-specific promoter was evaluated in HPV-negatives epithelial cells. The AD293 and C-33 A cells were co-infected with AdE2mIL-12 (50 MOIs) and of AdCMVE2HPV16 (50-100 MOI). Twenty hours after the AdE2mIL-12 infection, neither of the cells expressed the HPV E2 and p40 (IL-12) proteins. However, when the cells were infected with AdCMVE2HPV16, the induction of the p40 subunit of IL-12 was detected, and it was determined that it was relate to the expression of the HPV E2 protein in HPV-negatives cells and that it was dose-dependent (Fig. 3B). Furthermore, the in situ activity of HPV E2-specific promoter was evaluated in the in tumor-bearing mice injected intratumorally with co-administration of the adenovirus 
AdCMVE2HPV16 and AdE2IL-12 (Fig. 4). The p40 subunit of IL-12 was detected in tumor tissues 48 hours after the administration with both recombinant adenovirus and the detection was more evident that the mice treated with AdCMVmIL-12.

\section{Coinjection with AdE2IL-12 and} AdCMVE2HPV16 induces tumor inhibition in vivo. Coinjection of AdE2mIL-12 and AdCMVE2HPV16 into tumor-bearing mice produced a complete regression of tumor in the time period assessed. Approximately five days after treatment with both adenoviruses, reduction in tumor growth was observed (Fig. 5). There was no difference in tumor inhibition between the group of mice treated with the AdE2mIL-12 and AdCMVE2HPV16 coinjection and the group of mice treated with the AdCMVmIL-12 alone, in the tumor volume analyzed. On the other hand, the group of mice treated with
AdCMVE2HPV16 alone showed an inhibition of tumor growth, which was maintained up to 15 days after the injection, at which point tumor growth resumed although at a slower pace.

Survival curves of tumor-bearing mice treated with recombinant adenovirus. We detected a slight increase in the survival of mice treated with AdCMVGFP and AdE2mIL-12 adenoviruses compared to controls (Fig. 6). A survival advantage was detected in mice treated with AdCMVE2HPV16 (57\% more than control) but the greatest effect on survival was observed with treatment with AdCMVmIL-12 and co-injection of AdE2mIL-12 and AdCMVE2HPV16. Coinjection of both viral vectors had tumor inhibitory effects in 4 of $5(80 \%)$ mice, and we observed a significant survival of mice compared with the control group.
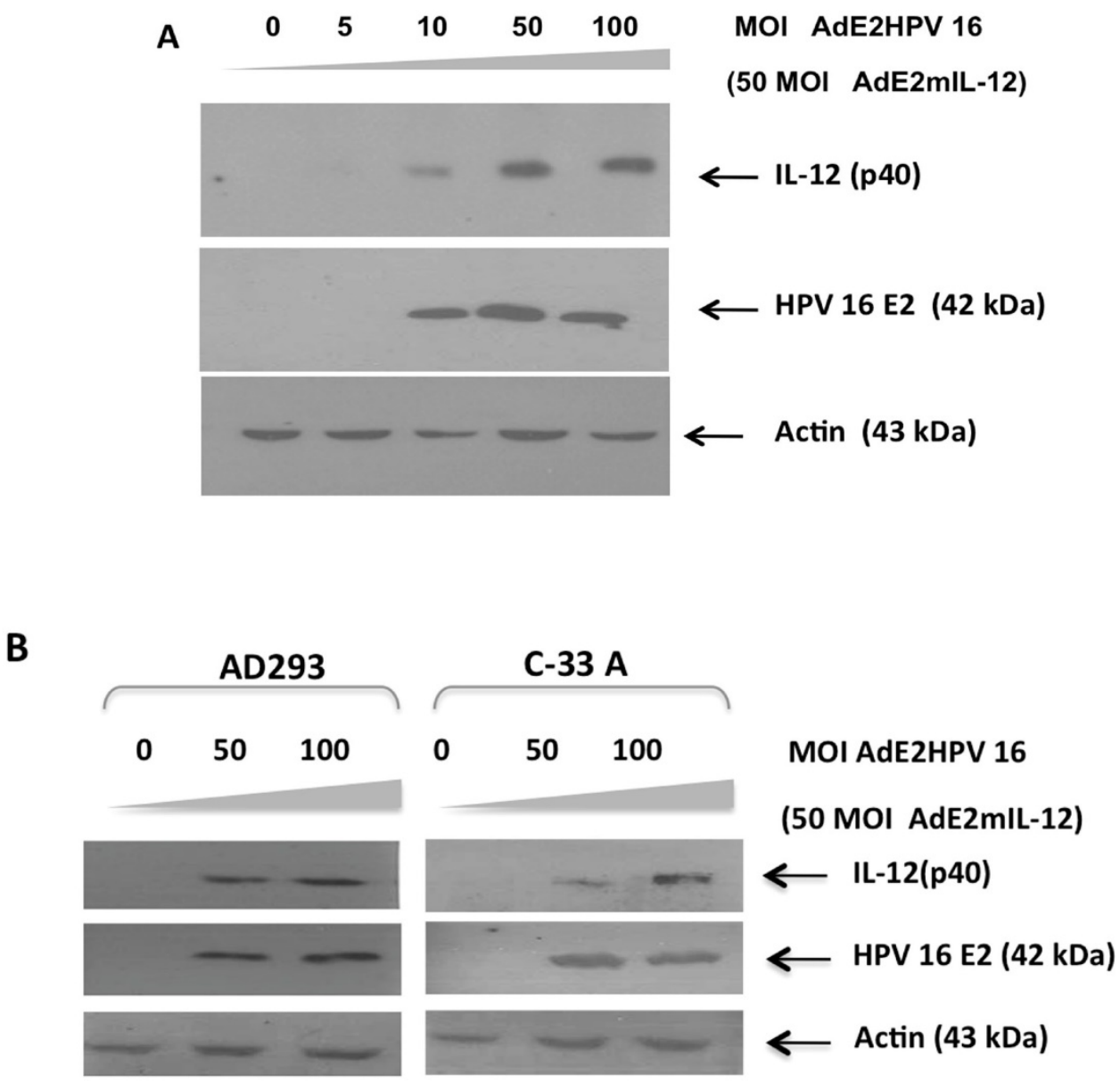

Figure 3: Transcriptional induction of IL-12 by HPV 16 E2 protein through the HPV E2-specific promoter. A). BMK-16/myc cells (HPV 16 positives) were co-infected with adenovirus AdE2mIL-12 (MOI 50) and with increasing doses of adenovirus AdCMVE2HPV 6 (5-100 MOI), 48 hrs after treatment, presence of mIL-12 protein was detected by western blot. B). HPV-negative AD293 and C-33A cells were co-infected with AdE2mIL-12 (50 MOls) and AdCMVE2HPV16 (50-100 MOI); 48 hrs after infection, HPV 16 E2 protein and $\mathrm{p} 40$ protein (IL-12) were detected the by Western blot. 


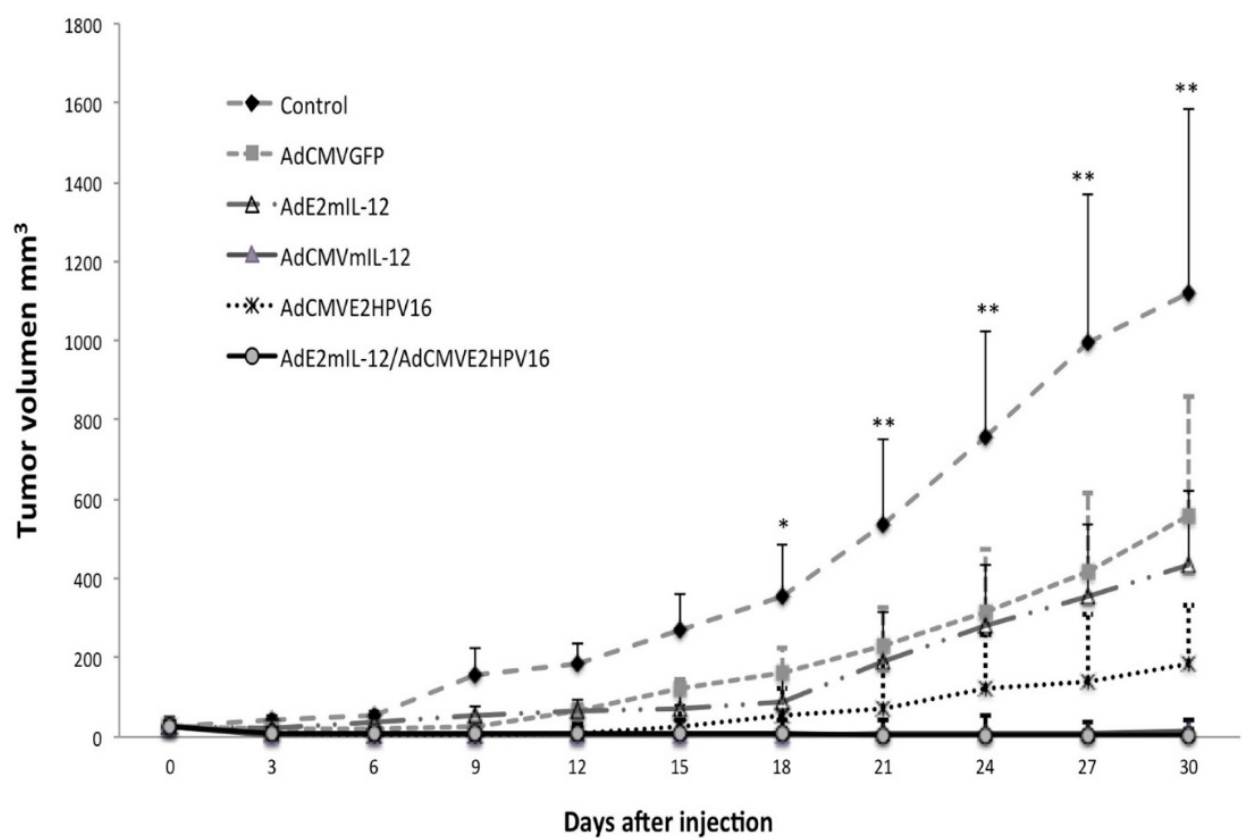

Figure 4: Antitumor Effect of HPV 16 E2 gene and IL-12 by recombinant adenoviral vectors evaluated in an HPV 16-positive murine tumor model. BALB / c mice with a tumor induced by administration of BMK-16 / myc cells were treated with recombinant adenoviral vectors AdCMVE2HPV16, AdCMVmIL-12, AdE2mIL-12, AdCMVGFP and co-administration of AdE2mlL-12 adenovirus and AdCMVE2HPV16. Subsequently, tumor growth was assessed at different over time. Data are presented as $* X \pm S E$. $P<0.0001$ compared between groups. Data are presented as $\mathrm{X} \pm \mathrm{SE}$. *P <0.05: AdCMVmIL-12 and AdCMVGFP versus controls. **P <0.01: AdCMVmlL-12 and AdCMVGFP versus controls (Student's t-test).

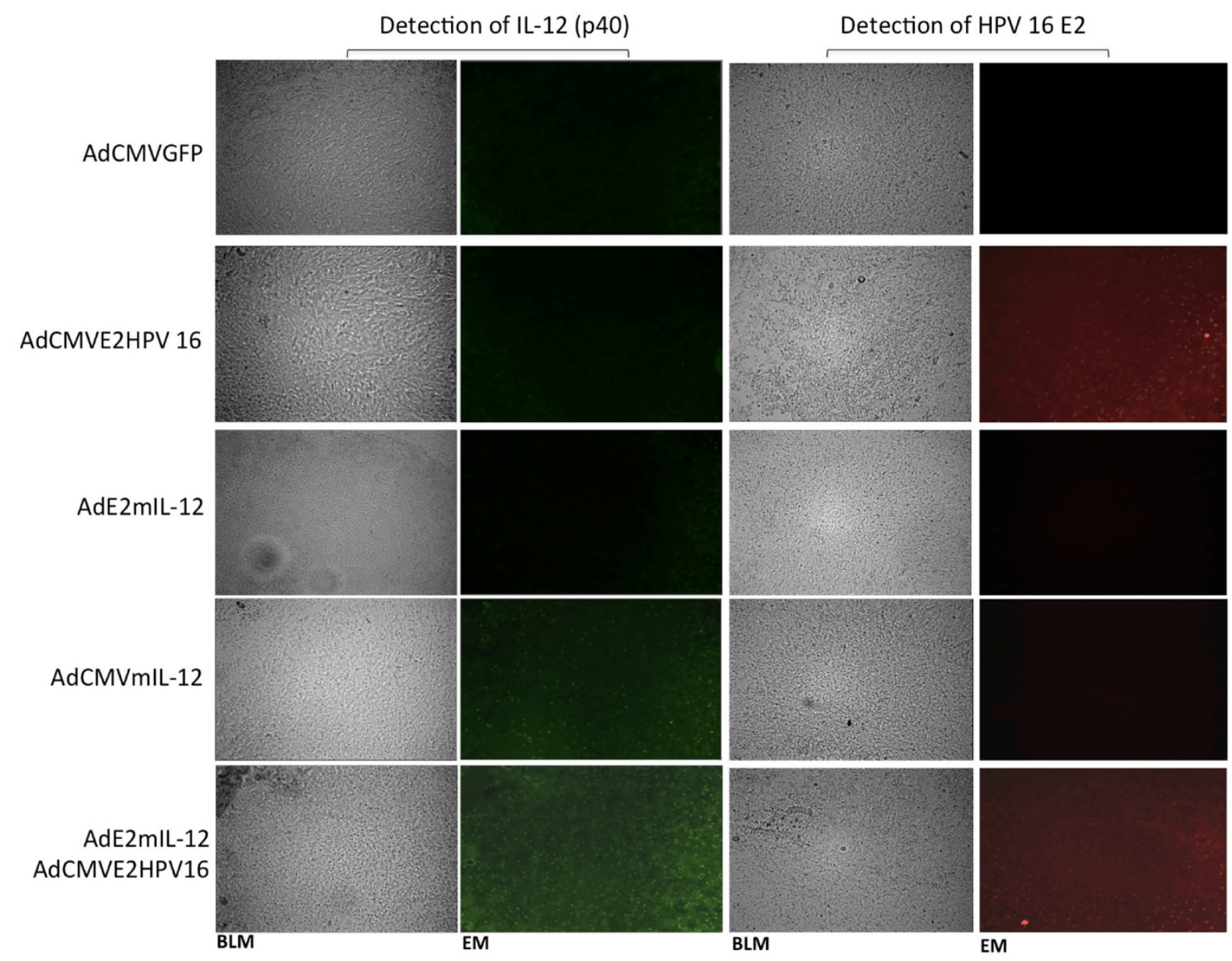

Figure 5: Detection of in situ of the proteins expressed by recombinant adenovirus and the activity of HPV E2-specific promoter. Tumor-bearing mice were injected intratumoral with $5 \times 10^{5}$ PFUs of recombinant adenovirus AdCMVE2HPV16, AdCMVmlL-12, AdE2mlL-12 and AdCMVGFP and was the proteins mIL-12 and HPV 16 E2 were detected by immunohistochemistry. Additionally, other group of tumor-bearing mice was injected with $5 \times 10^{3}$ PFU of each adenovirus (AdE2mIL-12, AdCMVE2HPV16) and the activity of HPV E2-specific promoter was evaluated. Magnification: X20. BLM: Bright field microscopy, EM: Epifluorescence microscopy. 


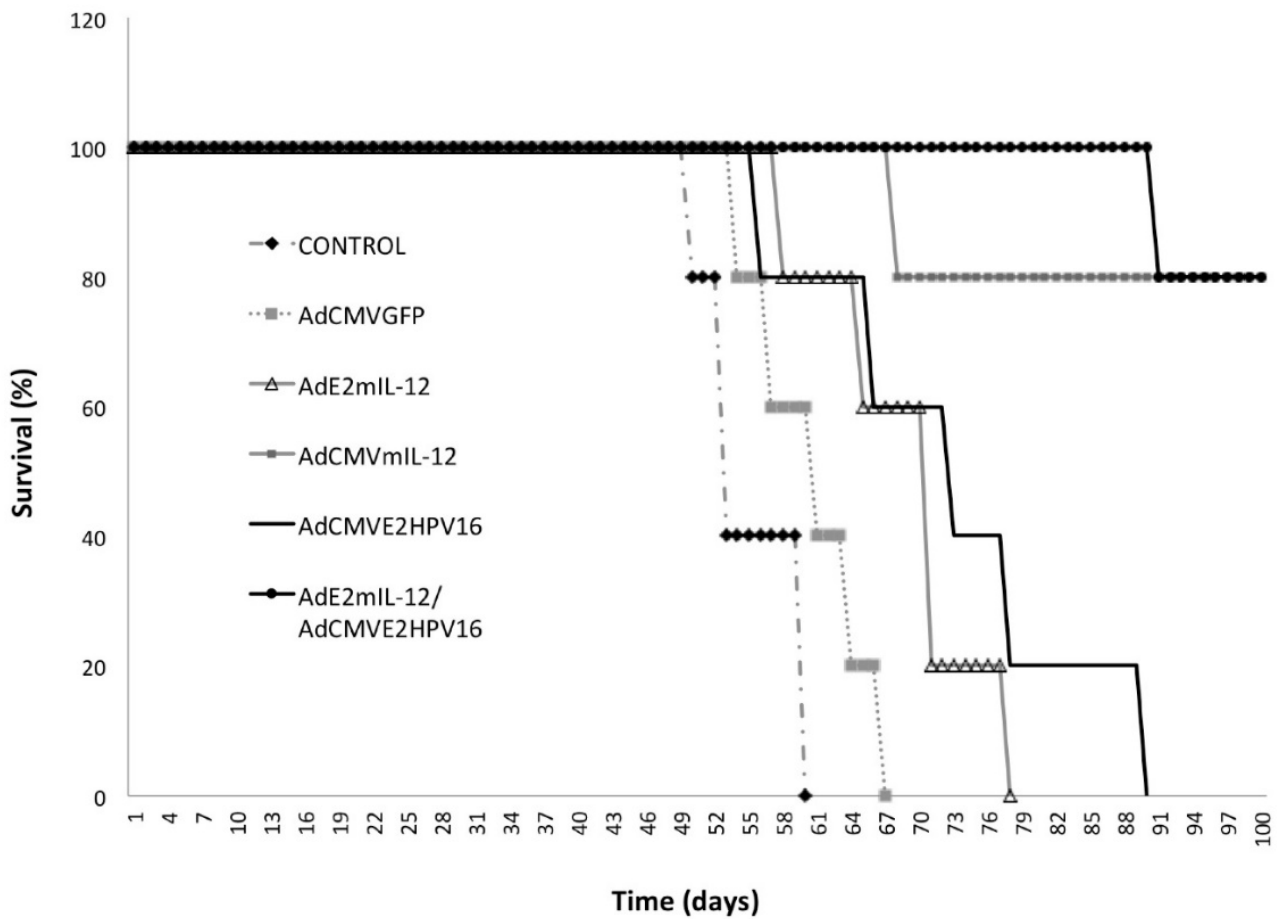

Figure 6: Survival of tumor-bearing mice treated with recombinant adenoviruses.

\section{Discussion}

In the present study, we demonstrate that in vivo application of an HPV E2-specific promoter to induce expression of IL-12 in HPV 16-positive tumor-bearing mice using viral gene therapy, lead to significant inhibition of tumor growth and increased survival in this murine tumor model.

In precancerous cervical lesions, the HPV E2 protein appears to play a role in the viral life cycle by regulating the transcription and replication of viral genes. ${ }^{14-16}$ These biological functions of E2 are mediated through the binding of a palindromic DNA sequence, $\mathrm{ACCN}{ }_{6} \mathrm{GGT}$, referred to as the E2 binding site (E2BS) in all papillomavirus. ${ }^{15-17}$ This observation led us to hypothesize that E2BS of HPV would be a candidate sequence to drive the expression of therapeutic genes and inhibit cancer development in precancerous cervical lesions. We found that an HPV E2-specific promoter that drives the expression of IL-12 had functional activity in vitro and in vivo mediated by the viral gene. In the in vitro system, we determined that the HPV E2-specific promoter is trans-activated by the HPV 16 E2 protein and is dose-dependent on HPV 16 E2 protein concentration. Additionally, we demonstrated that the HPV E2-specific promoter would not be activated in cells HPV-negatives (C-33 A), and that the presence of the HPV E2 protein was necessary to promote the activation. However, it is necessary to evaluate the HPV E2-specific promoter in other epithelial cells and in vivo HPV-negative systems. In our experimental system, we used the four E2 transcriptional elements with two SP1 transcriptional elements as a promoter to drive IL-12 expression by means of recombinant adenovirus. This technique stands in contrast with other systems that employ complete sequences in the HPV genome such as the LCR, which can be regulated by the E2 protein, or general transcription factors expressed in both HPV-infected and un-infected cells ${ }^{32}$.

In vivo analysis of the HPV E2-specific promoter's ability to induce IL-12 expression in tumor-bearing mice, demonstrated the promoter's functionality. Treatment was associated with the inhibition of tumor growth and increased survival in the HPV 16 positive murine tumor model. $80 \%$ of tumor-bearing mice coinjected with the adenoviral vectors AdCMVE2HPV16 and AdE2mIL-12 showed almost complete tumor suppression 30 days after injection. This result is significant, as the viral sequence (E2BS) may have potential therapeutic applications. On the other hand, we observed a partial antitumor effect after injecting the recombinant adenovirus in the animal tumor model. This effect was detected until 10 days after the administration, later the tumor growth depends on the effect of the therapeutic genes. It is documented that the single administration of the recombinant adenovirus to the tumor tissue favors the activation of specific immune responses, involving the recruitment and activation of inflammatory cells to viral antigens ${ }^{35-37}$. Therefore, the 
activation of the immune response depends of the production of the viral antigens, which is restricted by the replication incompetent adenovirus. The local immune response generated by the recombinant adenovirus, involving the expression and presentation of viral antigens by the tumor cells, together with the activation of $\mathrm{T}$ cells promotes the antitumor effect, but this effect is transitory. Also, an antitumor effect was observed when the tumor-bearing mice were treated with AdCMVE2HPV16, a significant reduction was observed in corporation with the control $(p<0.01)$. Previously, we shown the ability of the HPV 16 E2 protein (using the recombinant adenovirus AdCMVE2HPV16) inhibited cell growth and promote cell death by apoptosis in both human and murine HPV 16 transformed epithelial cells as well as we observed anti-tumor effects in vivo HPV 16 tumor-bearing mice. ${ }^{34}$ Nevertheless, the antitumor effect of the AdCMVE2HPV16 was lower than the one observed with AdCMVmIL-12 and with the co-administration of AdCMVE2HPV16 and AdE2mIL-12. The activation of immune response by the IL-12 protein produced by the AdCMVmIL-12 and AdE2mIL-12 has greater antitumor effect than the HPV 16 E2 protein produced by AdCMVE2HPV16. However, not differences in the antitumor effect were observed using AdCMVmIL-12 or co-administration of the AdCMVE2HPV16 and AdE2mIL-12. The production of the IL-12 protein by the adenoviruses induces the same antitumor effect using the same tumor volume; perhaps differences on growth tumor inhibition using the AdCMVmIL-12 or co-administration of the AdCMVE2HPV16 and AdE2mIL-1 can be observed in larger tumor. Which present a greater range to observed differences in the production of the IL-12 protein related with the increase to the tumor growth inhibition.

The antitumor effect of gene therapy using IL-12 has been demonstrated in a number of preclinical models in relation with the recombinant protein use. $^{21-24}$ However, most of these studies used ubiquitous promoters to regulate expression of the therapeutic genes, which causes low-specific toxicity to normal tissues. ${ }^{7,8}$ For this reason it is necessary to develop novel cancer-specific promoters selective for HPV-infected cells, to ensure expression of the therapeutic gene in infected cells and avoid gene activation in normal cells and tissues.

The HPV E2-specific promoter represents a sequence with potential therapeutic implications in cervical precursor lesions, ${ }^{38-40}$ at which point the HPV genome exists as an extrachromosomal element and the E2 protein is continuously expressed. However, in malignant cervical lesions, the chromosomal integration of HPV frequently results in disruption of the E2 open reading frame and consequent loss of E2 protein, 15,16 in which case the HPV E2-specific promoter would not be functional. The therapeutic application of the AdE2mIL12 in cervical precursor lesions, with the intrinsic expression of HPV E2 protein, could favor the activation of the cellular immune response in early stages and prevent progression malignancy. This therapeutic strategy will be applicable in cervical precursor lesions associated with someone HPV genotypes. All the papillomavirus express the E2 protein and interact with the E2BS, and will be could potentially activate the expression of IL-12 (of the AdE2IL-12) and stimulation of the cellular immune response. Furthermore, It is necessary to consider that the toxicity associated with the in vivo administration of adenovirus vectors and the pre-existing immunity to the prevalent adenovirus serotypes acquired as a result of natural infections, ${ }^{41}$ have the potential to modulate vector efficacy and safety. ${ }^{41-43}$ As these, vectors have been improved; the gene delivery technologies have rapidly evolved and thus it is important to investigate other options such as noviral biological agents, including liposomes, exosomes, ex-vivo gene transfer, DNA nanostructures, mesenchymal stem cells, and nano-biopolymers. ${ }^{44-49}$ Also, the systemic therapy for cancer carried out with IL-12 is associated with toxicity; therefore, the local application using gene therapy should results in a much higher local IL-12 concentration, and consequently a greater beneficial effect in terms of tumor immunity. ${ }^{24}$ Furthermore, combining a gene transfer with other breakthroughs from biomedical research and novel biotechnologies opens new avenues for the treatment of cervical cancer and precancerous lesions with gene therapy.

In summary, we have demonstrated that the HPV E2-specific promoter driving the expression of IL-12 has functional activity in vitro and in vivo, and has the capacity to significantly inhibit tumor growth in an HPV 16 positive murine tumor model. Further investigation is being carried out to optimize gene delivery methods, dosing and administration.

\section{Acknowledgments}

The funds for this work were obtained from the Mexican government through a Grand National Council of Science and Technology (CONACYT) with file numbers: Conacyt-Desarrollo Científico para Atender Problemas Nacionales 2013-215484. We thank to the National Advanced Microscopy Laboratory, Biotechnology Institute. National Autonomous University of Mexico, Cuernavaca Morelos, México. 


\section{Conflicts of Interest}

\section{The authors declare no conflict of interest.}

\section{References}

1. Muñoz N, Bosh FX, de Sanjosé S, Herrero R et al. Epidemiologic classification of human papillomavirus types associated with cervical cancer. N Engl J Med 2003; 348:518-527.

2. Palacio-Mejía LS, Lazcano-ponce E, Allen-Leigh B, Hernández-Avila M. Regional differences in breast and cervical cancer mortality in Mexico between 1979-206. Salud Publica Mex 2009;51:208-219.

3. Doorbar J. Molecular biology of human papillomavirus infection and cervical cancer. Clin Sci 2006;110:525-541.

4. Malcolm K, et al. Cancer Gene Therapy by Viral and Non-viral Vectors willey. Chapter 5: Cancer gene therapy by tisue-specific and cancer-taegetin promoters. 2014: 1045-1089.

5. Azzouz M. Gene Therapy for ALS: progress and prospects. Biochim Biophys Acta. 2006;1762:1122-1127.

6. Papadakis ED, Nicklin SA, Baker AH, et al. Promoters and control elements: designing expression cassettes for gene therapy. Curr Gene Ther 2004;4:89-1113.

7. Walther W, Stein U. Cell type specific and inducible promoters for vectors in gene therapy as an approach for cell targeting. J Mol Med (Berl) 1996;74:379-392.

8. Lin $\mathrm{T}, \mathrm{Gu} \mathrm{J}$, Zhang $\mathrm{L}$, Huang $\mathrm{X}$, et al. Targeted expression of green fluorescent protein/tumor necrosis factor-related apoptosis-inducing ligand fusion protein from human telomerase reverse transcriptase promoter elicits antitumor activity without toxic effects on primary human hepatocytes. Cancer Res 2002;62:3620-3625.

9. Roth JA, Cristiano RJ. Gene therapy for cancer: what have we done and where are we going?. J Natl Cancer Inst 1997;89:21-39.

10. Naidoo J, Young D. Gene regulation systems for gene therapy applications in the central nervous system. Neurol Res Int 2012;2012:1-8.

11. Thierry F. Transcriptional regulation of the papillomavirus oncogenes by cellular and viral transcription factors in cervical carcinoma. Virol 2009;384:375-379.

12. Peng W, Dunton C, Holtz D, et al. DNA nanotherapy for pre-neoplastic cervical lesions. Gynecol Oncol 2013;128:101-116.

13. Dukagjin $\mathrm{M}$, et al. Evolutionary and biophysical relationships among the papillomavirus E2 proteins. Front Biosci 2009;14:900-917.

14. Moskaluk C, Bastia D. The E2 gene: of bovine papillomavirus encodes an enhancer-binding protein. Proc Natl Acad Sci USA 1987;84:1215-1218.

15. Muller M, Demeret C. The HPV E2 E2-host protein-protein interactions: A complex hijacking of the celular network. Open Virol J. 2012;6:173-189.

16. McBride AA, Romanczuk H, Howley PM. The papillomavirus E2 regulatory proteins. J Biol Chem 1991;266:18411-18414.

17. Tierry F, Yaniv M. The BPV1-E2 transacting protein can be either an activator or a repressor pf the HPV 18 regulatory region. EMBO J 1987;6:3391-3397.

18. Del Vecchio M, Bajetta E, Canova S, et al. Interleukin-12: biological properties and clinical application. Clin Cancer Res 2007;13:4677-4685.

19. Xu HM. Th1 cytokine-based immunotherapy for cáncer. Hepatobiliary Pancreat Dis Int. 2014;13:482-494.

20. Berraondo P, Prieto J, Gonzalez-Aseguinolaza G. Advances in interldikin-12 gene therapy for acquired liver diseases. Curr Gene Ther 2009:9:62-71.

21. Nastala CL, Edington HD, McKinney TG, et al. Recombinant IL-12 administration induces tumor regression in associated with IFN$\gamma$ production. J Immunol 1994;153:1697-1706.

22. Rakhmilevich AL, Janssen $\mathrm{K}$, Hao Z, et al. Interleukin 12 gene therapy of a weakly immunogenic mouse mammary carcinoma results in reduction of spontaneous lung metastases via a $\mathrm{T}$ cell-independent mechanism. Cancer Gene Therapy 2000;7: 826-838.

23. Golab J, Zagozdzon R. Antitumor effects of interleukin-12 in pre-clinical and early clinical studies. Int J Mol Med 1999;3:537-544.

24. Mahvi DM, Henry MB, Albertini MR, et al. Intratumoral injection of IL-12 plasmid DNA--results of a phase I/IB clinical trial. Cancer Gene Ther 2007;14:717-723.

25. Torres-Poveda $\mathrm{K}$, Bahena-Román M, Madrid-González $\mathrm{C}$ et al. Role of IL-10 and TGF-B1 in local immunosuppression in HPV-associated cervical neoplasia. World J Clin Oncol 2014;5:753-63.

26. Ma W, Wang $\mathrm{K}, \mathrm{Du} J$, Luan $\mathrm{J}$, et al Multi-dose parecoxib provides an immunoprotective effect by balancing T helper 1 (Th1), TH2, TH17 and regulatory $\mathrm{T}$ cytokines following laparoscopy in patients with cervical cáncer. Mol Med Rep 2015;11:2999-3008.

27. Bermúdez-Morales VH, Peralta-Zaragoza O, Alcocer-González JM, et al. IL-10 expression is regulated by HPV E2 protein in cervical cancer cells. Mol Med Rep 2011;4:369-375

28. Feng $\mathrm{Q}$, Wei $\mathrm{H}$, Morihara J, et al. Th2 type inflammation promotes the gradual progression of HPV-infected cervical cells to cervical cells carcinoma. Gynecol Oncol 2012;127:412-419.

29. Sheu BC, Lin $\mathrm{RH}$, Lien $\mathrm{HC}$, et al. Predominant Th2/Tc2 polarity of tumor-infiltrating lymphocytes in human cervical cancer. J Immunol 2001;167:2972-2978.
30. Díaz-Benítez CE, Navarro-Fuentes KR, Flores-Sosa JA, et al CD3zeta Expression and T Cell Proliferation are Inhibited by TGF-beta1 and IL-10 in Cervical Cancer Patients. J Clin Immunol 2009;29:532-544.

31. Crook T, Almond N, Murray A, et al. Constitutive expression of c-myc oncogene confers hormone independence and enhanced growth-factor responsiveness on cells transformed by human papilloma virus type 16. Proc Natl Acad Sci U S A 1989:86:5713-5717.

32. Veress G, Szarka K, Dong X-P, et al. Functional significance of sequence variation in the E2 gene and the long control region of human papillomavirus type 16. J Gen Virol 1999;80:1035-1043.

33. He TC, Zhou S, da Costa LT, et al. A simplified system for generating recombinant adenoviruses. Proc Natl Acad Sci USA 1998;95:2509-2514.

34. Bermúdez Morales VH, Peralta Zaragoza O, Guzmán-Olea E, et al. HPV 16 E2 protein induces apoptosis in human and murine HPV 16 transformed epithelial cells and has antitumoral effects in vivo. Tumor Biol 2009;30:61-72.

35. Bessis N, Garcia-Cozar FJ, Boissier MC. Immune response to gene therapy vectors: influence on vector function and effectormechanisms. Gene Ther 2004;11:S10.17.

36. Gregory SM, Nazir SA, Metcalf JP. Implications of the innate immune response to adenovirus and adenoviral vectors. Future Virol 2011;6:357-374.

37. Ahi YS, Bangari DS, Mittal SK: Adenoviral vector immunity: its implications and circumvention strategies. Curr Gene Ther 2011; 11:307-320.

38. Giaretta A, Di Camillo B, Barzon L, et al. Modeling HPV early promoter regulation. Conf Proc IEEE ENG Med Biol Soc 2015;2015:6493-6496.

39. Lung MS, Mak WM, Murray V. The use of a human papilomavirus 18 promoter for tissue-specific expression in cervical carcinoma cells. Cell Mol Biol Lett 2011;16:477-492.

40. Sharma R. Palefsky JM. Addition of a single E2 binding site to the human papillomavirus (HPV) type 16 long control region killing of HPV positive cells via HPV E2 protein-regulated herpes simplex virus type 1 thymidine kinase-mediated suicide gene therapy. Human Gene Ther 2010;21:843-854.

41. Varnavski AN, Calcedo $\mathrm{R}$, Bove $\mathrm{M}$, et al. Evaluation of toxicity from high-dose systemic administrationof recombinant adenovirus vector in vector-naïve and pre-immunized mice. Gene Ther 2005;12:427-436.

42. Hunt KK, Vonbuger SA, Swisher SG. Gene Therapy to cancer. Charper 2: Efficacy, toxicity and immunogenicity of adenoviral vectors. By Shayakhetov DM. Humana Press. Totowa New Jersey. 2007:23-38.

43. Seow Y, Wood MJ. Biological gene delivery vehicles: beyond viral vectors. Mol Ther 2009;17:767-677.

44. Wang D, Gao G. State-of-the-art human gene therapy: part II. Gene therapy strategies and clinical applications: Discov Med 2014;18:151-161.

45. Porada CD. Almeida-porada G: Mesenchymal stem cells as therapeutics and vehicles for gene and drug delivery. Adv Drug Deliv Rev 2010;62:1156-1166.

46. Cheng Y, Wei H, Tan JK, et al. Nano-sezed sunflower polycations as affective gene transfer vehicles. Samall 2016;12:2750-2758.

47. Mokhtarzadeh A, Alibakhshi A, Yaghoobi H, et al. Recent advances on biocompatible and biodegradable nanoparticles as gene carriers. Exprt Opin Biol Ther 2016:16:771-785.

48. Lanier LA, Bermudez H. DNA nanostructures: a shift from assembly to applications. Curr Opin Chem Eng 2015;1:93-100

49. Willem DO, John JL, Jan J. et al. Local therapy of cancer with free IL-2. Cancer Immunol Immunother 2008; 57(7): 931-950.

50. Van der Jeught K, Bialkowski L, Daszkiewicz L, et al. Targeting the tumor microenvironment to enhancer antitumor immune responses. Oncotarget 2015; 6:1359-1381. 